\title{
Helmintos gastrointestinales zoonóticos de perros en parques públicos y su peligro para la salud pública
}

Gastrointestinal helminths zoonotic dogs in public parks and public health hazard

Gastrointestinal helmintos zoonóticas cães em parques públicos e de saúde pública perigo

Maritza Morales Sánchez' , Sebastián Soto Olarte', Zayra Cristina Villada Durango', Jhonny Alberto Buitrago Mejía', Natalia Uribe Corrales ${ }^{\circledR}$

Fecha correspondencia:

Recibido: octubre 11 de 2015.

Revisado: enero 28 de 2016.

Aceptado: marzo 4 de 2016.

Forma de citar:

Morales M, Soto S, Villada Z,

Buitrago J, Uribe N. Helmintos

gastrointestinales zoonóticos de perros en parques públicos y su peligro para la salud pública. Rev

CES Salud Pública. 2016; 7 (2)

\section{Open access}

(C) Copyright

Licencia creative commons

Ética de publicaciones

Revisión por pares

Gestión por Open Journal System

ISSN: 2145-9932

Sobre los autores:

1. Estudiantes, Grupo de investigación GINVER. Corporación Universitaria Remington. Medellín, Colombia.

\section{MVZ. Grupo de investigación} Epidemiología y Bioestadística. Universidad CES, Medellín, Colombia.

Comparte

\section{Resumen}

Los helmintos gastrointestinales zoonóticos que parasitan a los caninos representan un riesgo de salud pública; siendo los parásitos más reportados el Toxocara canis y el Ancylostoma, generando infecciones con sintomatología variada siendo el resultado de adaptación entre el parásito y su hospedero. En el mundo se han reportado diversos estudios sobre la presencia de estos parásitos en heces y suelos de lugares públicos, los cuales producen en el hombre sintomatología clínica, que se adquiere por el consumo accidental de estadíos larvarios que contaminan el suelo. Teniendo en cuenta la importancia que este tema demanda en la salud pública y cómo estos parásitos pueden afectar la salud humana y animal se realizó una revisión sistemática con el propósito de conocer los estudios que se han realizado en la temática, identificando los factores asociados a la infección animal que puedan contribuir al diseño de medidas de control.

Palabras clave: Helmintos, parásitos, zoonosis, perros, zonas de recreación.

\section{Abstract}

Zoonotic gastrointestinal helminths that parasitize the canine population represent a public health hazard. The principals parasites reported are Toxocara canis and Ancylostoma caninum, generating infections with symptoms varied being the result of adaptation between the parasite and its host. In the world we have reported several studies on the presence of parasites in feces and floors on public places, which produce the clinical symptomatology in man, acquired by the accidental consumption instars that contaminate the soil. Taking into account the importance of this theme on public health and how these parasites can affect human and animal health, a systematic review was conducted in order to know the studies that have been conducted on the subject, identifying the associated factors the animal infection that can contribute to the design of control measures.

Keywords: Helminths, parasites, zoonosis, dogs, recreation areas. 


\section{Resumo}

Helmintos gastrointestinais zoonóticas que parasitam caninos representar um risco para a saúde pública; sendo os parasitas relatou o Toxocara canis e Ancylostoma, gerando infecções com sintomas variados sendo o resultado de adaptação entre o parasita e seu hospedeiro. No mundo em que relataram vários estudos sobre a presença desses parasitas nas fezes e no solo de locais públicos, que ocorrem em sintomas clínicos homem, adquiridos pelo consumo acidental de estádios que contaminam o solo. Dada a importância desta demanda problema na saúde pública e como estes parasitas podem afetar a saúde humana e animal uma revisão sistemática foi conduzida de modo a conhecer os estudos que têm sido realizados sobre o assunto, identificando fatores associados infecção animal que pode contribuir para a concepção de medidas de controle.

Palavras chave: Helmintos, Parasitos, Cães, Zonas de Recreação.

\section{Introducción}

Los parásitos son microorganismos unicelulares o pluricelulares que viven a expensas de otro ser causándole perjuicio, que en su mayoría necesitan de organismos específicos para su supervivencia; en medicina, este nombre se ha reservado a los protozoos y los helmintos que viven temporal o permanentemente en el hombre, siendo la mayoría patógenos (1- 1 ).

Con el término helminto se hace referencia a los animales invertebrados que poseen aspecto de gusanos, órganos definidos, sin extremidades, con reproducción sexuada en el estadío adulto y de tamaño variable, el cual oscila desde pocos milímetros hasta metros. Estos parásitos pueden dividirse en tres grupos: los anélidos o gusanos segmentados, los platelmintos o gusanos planos y los nematelmintos o gusanos redondos ()ㅡ.

En los caninos, los helmintos afectan principalmente el tracto gastrointestinal y son un riesgo para la salud humana; siendo importante el estudio de sus ciclos de vida debido a que sus estadíos inmaduros son eliminados en las heces siendo la fuente de contaminación de suelos, a partir de los cuales se podrían infectar los animales y el hombre al ingerir alimentos, agua contaminada, o ingresando vía percutánea, de esta manera, el inadecuado manejo de excretas de la población canina visitante de parque públicos incrementa el riesgo de transmisión de estos patógenos (므).

Una vez las personas han sido contaminadas por la vía oral, las larvas son liberadas en el intestino y pasan a circulación sistémica desde donde migran hacia diversos órganos y tejidos generando lesiones traumáticas y algunos daños derivados de la respuesta inmune provocada por los antígenos parasitarios (1, $\underline{6}-\underline{11})$.

Múltiples estudios en el mundo han resaltado el impacto de los nemátodos en la salud publica debido a que ocasionan enfermedades tales como el síndrome de larva migrans visceral (Toxocara canis) y síndrome de larva migrans cutánea (Ancylostoma sp) y algunas enfermedades gastroentéricas; y resaltan que las plazas y parques públicos son lugares que actúan como reservorio de infecciones helmínticas, en Colombia se han encontrado algunos reportes en ciudades como $(\underline{5}-\underline{7}, \underline{\underline{1}}, \underline{11})$.

\section{Los caninos como reservorios de parásitos zoonóticos}

Los caninos pueden transmitir diversas especies de helmintos zoonóticos tales como Ancylostoma spp, Toxocara spp. Trichuris vulpis, Spirocerca spp, Uncinaria sp, 
Strogyloides spp, entre otros, los cuales pueden ocasionar en los humanes diversas patologías cutáneas, viscerales, oculares y cerebrales $(\underline{1}, \underline{6}, \underline{8}, \underline{9}, 11)$; ya que las excretas de los animales de compañía son un material biológico altamente contaminante de los alimentos, el agua y el suelo, los cuales constituyen las principales vías de trasmisión $(\underline{1,10})$.

La importancia de los caninos como fuente de infecciones parasitarias zoonóticas se ha demostrado en múltiples estudios como los realizados por Rojekittikhun en el 2014 en Tailandia, en un refugio de la ciudad de Nakhon Nayok, en el cual demostró que los animales de compañía actúan como hospedadores para varias especies de protozoos y helmintos gastrointestinales zoonóticos (12). De igual manera, en la India se encontró asociación significativa entre la presencia de Giardia en seres humanos con la presencia de caninos positivos a este parásito ( $P=0,0000$; IC del $95 \%$, $1,11 ; \underline{8}, \underline{13})$. En el 2012 otro estudio realizado en la ciudad de Teherán, sobre la presencia de parásitos zoonóticos en muestras de suelo de lugares públicos, concluye que los hallazgos sobre la contaminación del suelo con estos parásitos desempeñan un papel importante en la transmisión de enfermedades parasitarias zoonóticas al humano. Además se hace necesario un control de la población de animales como perros y gatos para reducir la distribución de los parásitos $(\underline{14,15})$.

\section{Riesgo para la salud pública}

En la actualidad la tenencia de mascotas se ha incrementado en las diversas sociedades y se ha asociado principalmente a factores emocionales y de seguridad, siendo los caninos la especie más utilizada para suplir estas necesidades, incrementando con ello el riesgo para la salud pública, principalmente en cuanto a la trasmisión de algunos parásitos zoonóticos, especialmente en la población infantil, haciendo necesario concientizar a la población acerca del adecuado cuidado y aseo de sus animales $(\underline{5}, \underline{10}, \underline{16}-\underline{19})$.

Uno de los principales helmintos al cual se le ha atribuido potencial zoonótico es el Toxocara canis, pues se ha asociado a diversos síndromes como la larva migrans visceral (VLM), larva migrans ocular (OLM), y la Toxocariosis encubierta (TC) (20), su importancia ha sido documentada en estudios realizados en los Países Bajos, en donde se encontró que casi el $50 \%$ de los casos Toxocariosis clínicas se asociaron con Toxocara canis y a los reportes realizados por Ehrhard y Kernbaum, quienes mediante una revisión sistemática evidenciaron que más de la mitad de los pacientes atendidos por Larva Migrans Visceral eran menores de tres años, concluyendo que este patógeno afectaba principalmente a infantes entre 1 y 3 años de edad, situación que fue asociada al mayor contacto de esta población con los caninos debido a una mayor frecuencia de juego y a los hábitos de geofagia de algunos de estos individuos (21).

Respecto a las infecciones causadas por Ancylostoma caninum, se le atribuyen la mayoría de las dermatitis parasitarias debido a su trasmisión percutánea en la cual causa una herida vulnerable a infecciones secundarias, y ocasiona una respuesta inflamatoria, adicionalmente se le ha asociado a problemas respiratorios como neumonías debido a que realiza migraciones cardiopulmonares (22-24). En la Universidad Central "Marta Abreu" de Las Villas, Cuba, se evaluó la presencia de nematodos zoonóticos como Toxocara canis y Ancylostoma caninum, siendo este último parásito el más prevalente y de más amplia distribución geográfica, siendo esto importante debido a que se facilita su diseminación en el medio ambiente (25). 


\section{Prevalencia de helmintos zoonóticos en lugares públicos}

En el mundo se han realizado estudios en los cuales se ha reportado la contaminación del suelo con diversas especies de parásitos zoonóticos, con una intensidad que varía en función de las características de la población humana, condiciones ambientales, tipo de urbanización y la población canina presente en cada uno de los lugares estudiados (21).

En Europa Stojcevic en el 2010 reporto una prevalencia del 15,5\% para Toxocara spp en parques públicos de Croacia (21); en Polonia en el 2012 se encontró en parques infantiles una contaminación con huevos de helmintos intestinales distribuida de la siguiente manera: Toxocara spp 73,5\%; Uncinaria 14,3\%; Ancylostoma 14,3\%; Ascaris spp 14,3\%; y Trichuris spp 4,1\% (26); en Madrid, en el 2012 se identificó una infección de Toxocara spp del $16,4 \%$ y Ancylostoma del $3 \%$, en muestras fecales de perros y gatos obtenidas del suelo de parques públicos (27); en Praga, la presencia de huevos de Toxocara spp fue del 45,0\% en muestras fecales de caninos en lugares públicos (28); en Nápoles en el 2006 el 16,9\% de heces caninas fueron positivos a Toxocara canis, Toxicara leonina, Ancylostoma caninum y Trichuris vulpis (29); y en Marche, Italia, se encontró Toxocara canis en un 33,6\% y se estableció que la positividad a estos parásitos era mayor en los caninos que habitaban el área rural con un $48,4 \%$ en comparación con los perros habitantes de la ciudad con una positividad del $26,2 \%$ (30).

En Asia en la ciudad de Kuala Lumpur, Noor para el 2008 estableció la prevalencia de Toxocara spp en un 12,1\% (31); por otro lado en parques públicos de Erzurum, Turquía se llevó a cabo una encuesta para determinar la presencia de huevos de Toxocara spp, cuyos resultados fueron un 64,26\% de Toxocara spp, y un 1,43\% para huevos del mismo parásito, lo que supone que los parques infantiles públicos deben ser cercados para evitar la contaminación fecal (르).

En América Latina, países como Perú, han demostrado que sus parques públicos constituyen zonas de riesgo para la zoonosis ocasionada por Toxocara canis (33), reportando prevalencia de huevos del $73,8 \%$ en muestras de suelo y del $57,6 \%$ en muestras de césped, concordando con los hallazgos obtenidos en otros países como Ecuador en donde se encontró una prevalencia de nemátodos del 13.6\% en Ciudad de Cuenca (34); y se concluyó que los parques de la zona urbana y rural son fuentes de contagio de enfermedad parasitaria, que podrían afectar a la salud de los seres humanos y a la salud de las mascotas (35), también países como Argentina, han obtenido prevalencias para Ancylostoma caninum y Trichuris vulpis del 62,96\%; Uncinaria stenocephala de 24,07\% y para Toxocara canis de 22,22\% (17); adicionalmente, en México se ha documentado como la contaminación con huevos de Toxocara canis tiene relevancia epidemiológica, pues al no realizar la recolección de la materia fecal en forma frecuente los huevos de este parásito quedan libres, ocasionando una dispersión continua con lo que se favorece la posible inhalación y deglución de los huevos y la infección en personas que laboran y habitan alrededor de estas áreas (36).

En Colombia se han realizado estudios en diversas zonas del país, encontrando en Bogotá D.C una prevalencia de helmintos gastrointestinales en caninos callejeros del $50 \%$ para Ancylostoma y del $23.86 \%$ para Toxocara canis (37); en Quindío se encontró una prevalencia del 13,9\% para Ancylostoma caninum, 4,3\% Trichuris vulpis; 2,5\% Toxocara canis y 4,0\% para Strongyloides stercoralis (9,19). En Popayán, se encontró una carga de Toxocara canis del 4,3\%. En la ciudad de Bucaramanga, considerando el estrato socioeconómico se evaluó la presencia de huevos de nemátodos zoonóticos encontrando que en los estratos altos (5 y 6) tienen una mayor prevalencia de Unci- 
naria spp con un 28,57\%, para el estrato medio (3 y 4) la prevalencia fue del 16, 67\% para Uncinaria spp y del 2,78\% para Toxocara y para el estrato bajo (1 y 2) los huevos de Toxocara spp presentaron una prevalencia del 10\% (․,11).

\section{Conclusiones}

Los helmintos zoonóticos que afectan el intestino de los canidos domésticos constituyen un alto riesgo para la salud publica especialmente cuando se tiene un pobre manejo sanitario de los animales y prácticas inadecuadas en el manejo de sus excretas, especialmente cuando estos son llevados a lugar públicos para realizar sus deposiciones; este tipo de prácticas ponen en riesgo principalmente a la población infantil debido a su mayor tendencia a algunas actividades como el juego con las mascotas, el contacto con suelos y pastos, y su predisposición a la geofagia. Esta situación hace necesario el desarrollo de políticas públicas para el control epidemiológico del parasitismo en parques públicos, el control de animales en condición de abandono y el fomento en la población acerca de la tenencia responsable de mascotas, la implementación de medidas sanitarias como la recolección de las excretas y la implementación de planes estratégicos de desparasitación.

\section{Bibliografía}

1. Rodríguez R, Cob L, Domínguez J. Frecuencia de parásitos gastrointestinales en animales domésticos diagnosticados en Yucatán. Rev Bioméd. 2001; 12:19-25. http://www.revbiomed.uady.mx/pdf/rb011214.pdf

2. Quiroz Romero H. Introducción al estudio de los parásitos: morfología, fisiología, nomenclatura y clasificación. In: Parasitología y Enfermedades Parasitarias de Animales Domésticos. 1st ed. México: Limusa S.A.; 1984. p. 876-876-894. http://www.laleo.com/parasitologia-enfermedades-parasitarias-de-animales-domesticos-p-1574.html

3. Prats G. Microbiología Clínica. In: Parasitología: Parásitos Humanos. 1st ed. Madrid, España: Panamericana; 2005. p. 127-400.

4. Negroni M. Microbiología histopatológica: fundamentos y guía práctica. In: Parásitos Generalidades. 2nd ed. Buenos Aires, Argentina: Panamericana; 2009. p. 95. http://www.medicapanamericana.com/Libros/Libro/4170/Microbiologia-Estomatologica.html

5. Devera R, Ytalia B, Amaya I, Nastasi MJ, Rojas G, Vargas B. Parásitos intestinales en habitantes de la comunidad rural "La Canoa", Estado Anzoátegui, Venezuela. Rev Venez Salud Pública. 2014; 2(1):15-21. dialnet

6. Caraballo A, Jaramillo A, Loaiza J. Prevalencia de parásitos intestinales en caninos atendidos en el Centro de Veterinaria y Zootecnia de la Universidad CES. Rev CES Med Vet Zootec. 2007; 2(2):24-31. http://revistas.ces.edu.co/index.php/ mvz/article/viewFile/375/1877

7. Cala Centeno FA, Durán López LF, Gómez Garzón C. Determinación de la presencia de estados inmaduros (huevos, larvas) de parásitos nematodos zoonóticos (Toxocara spp., Uncinaria spp. y Strongyloides spp.) en los parques públicos urbanos del municipio de Bucaramanga, Santander. Spei Donus. 2010;6(12):27-31. http://wb.ucc.edu.co/sdmvz/files/2013/06/articulo-3-vol-6-n-12.pdf 
8. Mendoza I, Callejas E, Hernández A, López J. Estudio comparativo de las parasitosis entéricas en las diferentes razas de perros diagnosticados en el Departamento de Parasitología. Vet México. 1993;24(4):335-7. http://new.medigraphic. com/cgi-bin/resumen.cgi?IDARTICULO $=15138$

9. Giraldo MI, García NL, Castaño JC. Prevalencia de helmintos intestinales en caninos del departamento del Quindío María Isabel Giraldo, Nora Lizeth García, Jhon Carlos Castaño. Biomédica [Internet]. 2005 [cited 2015 Sep 14];25(3). Available from: http:// www.scielo.org.co/scielo.php?pid=S0120-41572005000300010\&script=sci arttext

10. Milano A, Oscherov E. Contaminación de playas de la ciudad de corrientes con parásitos caninos capaces de infectar al hombre. [Internet]. Facultad de Ciencias Exactas y Naturales y Agrimensura; 2002 [cited 2015 Sep 10]. Available from: http://www.unne.edu.ar/unnevieja/Web/cyt/cyt/2001/6-Biologicas/B-036.pdf

11. Vásquez R, Campo V, Vergara D, Rivera O, Cordero H. Prevalencia de Toxocara canis y otros parásitos intestinales en caninos de la ciudad de Popayán, 2004. Centro de estudios en microbiología y parasitología - CEMPA; 2005. https:// www.researchgate.net/publication/237401615_PREVALENCIA_DE_Toxocara canis Y OTROS PARASITOS INTESTINALES EN CANINOS EN LA CIUDAD DE POPAYAN 2004

12. Rojekittikhun W, Chaisiri K, Mahittikorn A, Pubampen S. Gastrointestinal parasites of dogs and cats in a refuge in nakhon nayok thailand. Dep Protozool Fac Trop Med Mahidol Univ. 2014;45(1). https://www.ncbi.nlm.nih.gov/pubmed/24964651

13. Traub RJ, Robertson ID, Irwin PJ, Mencke N, Thompson RCAA. Canine gastrointestinal parasitic zoonoses in India. Trends Parasitol. 2005 Jan;21(1):42-8. https://www.ncbi.nlm.nih.gov/pubmed/15639740

14. Tavalla M, Oormazdi H, Akhlaghi L, Razmjou E, Moradi L. Prevalence of parasites in soil samples in Tehran public places 33. Tavalla M, H, L, E, Moradi LM. Afr J Biotechnol. 2012;11(20):4575-8. http://www.ajol.info/index.php/ajb/article/view/101348

15. Zibaei M, Abdollahppur F, Birjandi M, Firoozeh F. Soil contamination spp. eggs in the public parks from three áreas of Khorram Abad, Irán. Khorram Abad, Irán. Nepal Med J. 2010;12(2):12-6. https://www.ncbi.nlm.nih.gov/pubmed/21222397

16. Rubel D, Wisnivesky C. 31. Rubel D, Wisnivesky C. 2010. Contaminación fecal canina en plazas y veredas de Buenos Aires, 1991-2006. Medicina (B. Aires); Vol. 70 (4). Med B Aires. 2010;70(4):355-63. http://www.scielo.org.ar/scielo.php?scrip-

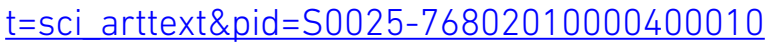

17. Andresiuk MV, Denegri GM, Esardella NH, Hollmann P. Encuesta coproparasitológico canina realizado en plazas publicas de la ciudad de Mar del Plata, Buenos Aires, Argentina. Parasitol Latinoam. 2003 Jan;58(1-2):17-22. http://www.scielo. $\underline{\mathrm{cl} / \text { scielo.php?script }=\text { sci arttext\&pid=S0717-77122003000100003 }}$

18. Andresiuk MV, Rodríguez F, Denegri GM, Sardella NH, Hollmann P. Relevamiento de parásitos zoonóticos en materia fecal canina y su importancia para la salud de los niños. Arch Argent Pediatría. 2004 Oct;102(5):325-9. http://www.scielo. org.ar/scielo.php?script=sci arttext\&pid=S0325-00752004000500003 
19. Solarte - Paredes L, Castañeda - Salazar R, Pulido - Villamarín A. Parásitos gastrointestinales en perros callejeros del centro de zoonosis de Bogotá D.C., Colombia. Neotropical Helminthol. 2013;7(1):25-31.

20. Overgaauw P, Van Knapen F. Overgaauw PA, van Knapen F. 2013. Veterinary and public health aspects of Toxocara spp Europa. Veterinary parasitology, Volume 193, Issue 4, 15 April 2013, Pages 398-403. Vet Parasitol. 2013;193(4):398-403. http://www.sciencedirect.com/science/article/pii/S0304401712006838

21. Stojcevic D, Susic V, Lucinger S. Contamination of soil and sand with parasite elementsas a risk factor for human health in public parks and playgrounds in pula, croatia. Vet Arch. 2010;80(6):324-7. http://www-staro.vef.unizg.hr/vetarhiv/papers/2010-80-6-5.pdf

22. Alipour H, Goldust M. Apparent contact dermatitis caused by Ancylostoma caninum: a case report. Ann Parasitol. 2015;61(2):125-7. https://www.ncbi.nlm. nih.gov/pubmed/26342510

23. McGrath I, Piliouras P, Robertson I. Irritant bullous contact dermatitis caused by a rove beetle: an illustrated clinical course. Australas J Dermatol. 2013;54:136-8. https://www.ncbi.nlm.nih.gov/pubmed/23581998

24. Mimesh S, Pratt M. Allergic contact dermatitis from corticosteroids: reproducibility of patch testing and correlation with intradermal testing. Dermatol J. 2006;17:137-42. https://www.ncbi.nlm.nih.gov/pubmed/16956466

25. César CCJ. Prevalencia y factores que favorecen la presentación de toxocara canis y ancylostoma caninum en canes de compañía (Prevalence and factors that favor of presentation of toxocara canis and ancylostoma caninum in companion dogs). 2012. http://www.veterinaria.org/revistas/redvet/n060612B/PR10.pdf

26. Blaszkowska J, Wojcik A, Kurnatowski P, Szwabe K. Geohelminth egg contamination of children's play areas in the city of Lodz (Poland). Vet Parasitol. 2013 Feb 18;192(1-3):228-33. https://www.ncbi.nlm.nih.gov/pubmed/23084538

27. Dado D, Izquierdo F, Vera O, Montoya A, Mateo M, Fency S, et al. Detection of zoonotic intestinal parasites in public parks of Spain. Potential epidemiological role of microsporidia. Zoonoses Public Health. 2012;59(1):23-8. https://www.ncbi. nlm.nih.gov/pubmed/21824364

28. Dubná S, Langrová I, Jankovská I, Vadlejch J, Pekár S, Nápravník J, et al. Contamination of soil with Toxocara eggs in urban (Prague) and rural areas in the Czech Republic. Vet Parasitol. 2007 Mar 15;144(1-2):81-6. https://www.ncbi.nlm. nih.gov/pubmed/17049747

29. Rinaldi L, Biggeri A, Carbone S, Musella V, Catelan D, Veneziano V, et al. Canine faecal contamination and parasitic risk in the city of Naples (southern Italy). BMC Vet Res. 2006;2:29. http://bmcvetres.biomedcentral.com/articles/10.1186/1746-6148-2-29

30. Habluetzel A, Traldi G, Ruggieri S, Attili AR, Scuppa P, Marchetti R, et al. An estimation of Toxocara canis prevalence in dogs, environmental egg contamination 
and risk of human infection in the Marche region of Italy. Vet Parasitol. 2003 May 1;113(3-4):243-52. http://www.sciencedirect.com/science/article/pii/ $\underline{\mathrm{S} 0304401703000827}$

31. Noor A, Sakhone L, Lokman S, Hakim M, Yussi Y. DETECTION OF HELMINTH INFECTIONS IN DOGS AND SOIL CONTAMINATION IN RURAL AND URBAN AREAS. Southeast Asian J Trop Med Public Health. 2008;39(2):205-12. https://www. ncbi.nlm.nih.gov/pubmed/18564703

32. Avcioglu H, Balkaya I. The relationship of public park accessibility to dogs to the presence of Toxocara species ova in the soil. Vector Borne Zoonotic Dis Larchmt N. 2011 Feb;11(2):177-80. https://www.ncbi.nlm.nih.gov/pubmed/20569014

33. Lannacone J, Alvariño L, Cárdenas-Callirgos J. CONTAMINACIÓN DE LOS SUELOS CON HUEVOS DE TOXOCARA CANIS EN PARQUES PÚBLICOS DE SANTIAGO DE SURCO, LIMA, PERÚ, 2007-2008. Neotropical Helminthol. 2012;6(1):97-109.

34. Ramón G. Prevalencia de helmintos gastrointestinales (cestodos y nemátodos) en caninos de la ciudad de cuenca [Internet] [Tesis de pregrado]. [Cuenca]: Universidad de Cuenca; 2012 [cited 2015 Sep 9]. Available from: http://dspace. ucuenca.edu.ec/bitstream/123456789/383/1/TESIS.pdf

35. Moreira N, Solórzano M. Determinación de huevos de parásitos intestinales de perros y gatos en el suelo de parques del Cartón Puerto Viejo en el año 2009. Escuela Superior Politécnica Agropecuaria de Manabí; 2010.

36. Martínez-Barbosa I, Cárdenas G, Marcia E, Sosa A, Arturo E, Lastra P, et al. Contaminación parasitaria en heces de perros, recolectadas en calles de la ciudad de San Cristóbal de Las Casas, Chiapas, México. Vet México. 2008 Jun;39(2):173-80. http://www.scielo.org.mx/scielo.php?script=sci arttext\&pi$\mathrm{d}=$ S0301-50922008000200006

37. Polo Terán L, Cortés Vecino J, Villamil Jiménez L, Prieto L. Contaminación de los parques públicos de la localidad de Suba, Bogotá con nematodos zoonóticos. Rev Salud Pública. 2007:9:534-40. http://www.scielosp.org/pdf/rsap/v9n4/ v9n4a07.pdf 